\title{
Sudden Unexpected Death in Epilepsy: What Would Darwin Say About It?
}

\author{
Carla Alessandra Scorza $\mathrm{PhD}^{1}$, Fulvio Alexandre Scorza $\mathrm{PhD}^{1}$, Josef Finsterer $\mathrm{MD}^{2 *}$ \\ ${ }^{\mathrm{T}}$ Disciplina de Neurociência Universidade Federal de São Paulo/Escola Paulista de Medicina (UNIFESP/EPM) São \\ Paulo, Brasil \\ ${ }^{2}$ Krankenanstalt Rudolfstiftung, Vienna, Messerli Institute, Vienna, Austria
}

*Corresponding Author

Josef Finsterer, MD, PhD

\author{
Article History \\ Received: 27.01 .2020 \\ Accepted: 03.02.2020 \\ Published: 09.02.2020
}

Keywords: JAMA Neurology, cognitive disorders, pileptologists, medical and scientific compassion.

\section{LETTER TO THE EDITOR}

Always on the lookout for Editorials and articles from JAMA Neurology, one in particular has attracted a lot of attention because these comments are really fascinating. Devinsky et al. [1] address some priority issues in clinical research related to the high rates of premature deaths in epilepsy, affective and cognitive disorders. We are in a period of much technology and little "medical and scientific compassion."

In this sense, the pertinent comments by Devinsky et al.[1] are stimulating, since the works carried out in the nineteenth century by Bacon[2] and the modern ones by Sveinsson[3], show that mortality in epilepsy exists (independent of the time and specific cause), but the emotions of patients and their intrapsychic dynamics are often neglected by epileptologists. It is worth noting that not even Bacon [2] and Sveinsson [3] - and we can include ourselves here as well - did not pay much attention to the fascinating work of Sir Charles Darwin from 1872, "The Expression of the Emotions in Man and Animals [4]".

Briefly, Drawin[4] portrays in his book an unprecedented insight into human emotion and its relation to the reactions present in other species, establishing an anatomical and functional relationship. Thus, are psyschiatric disorders challenging the expectations of natural selection? Perhaps, yes. Psychiatric comorbidities are common in epilepsy and are crucial modifiable risk factors, which can reduce death in epilepsy [5]. Despite the great advances in translational research evaluated with epilepsy-related-death today (including SUDEP)-from molecular aspects to possible preventive measures- one of the key issues is still missing: which is the patient's mental and emotional state?

Thus, the first point here would be to educate us, that is, to make a critical evaluation of how we should assess the emotional profile of an individual with epilepsy. In addition, addressing the same profile of family members and/or caregivers, since they are the individuals who are in the "day to day" with them and obviously, share the pains and delights of life with the patients. Is this patient, family member or caregiver emotionally prepared to hear about mortality in epilepsy (SUDEP or even other causes of death)? Have we been trained, or better yet, are we adequately training our multidisciplinary group (especially younger neurologists) to discuss this matter properly with these people? In addition of treating individuals with epilepsy, how to treat family members and caregivers? What are the best drugs to treat psychiatric comorbidities (so severe) in patients with epilepsy? What are the side effects of these drugs and their relationship with increased mortality in an individual with epilepsy?

Copyright @ 2020: This is an open-access article distributed under the terms of the Creative Commons Attribution license which permits unrestricted use, distribution, and reproduction in any medium for non commercial use (NonCommercial, or CC-BY-NC) provided the original author and source are credited. 
Anyway, while these and other "simple" questions remain unsolved, we must always reflect on the famous phrase of Charles Darwin: "Ignorance more frequently begets confidence than does knowledge: it is those who know little, not those who know much, who so positively assert that this or that problem will never be solved by science".

\section{REFERENCES}

1. Devinsky, O., Singh, A., Friedman, D. (2018). Deaths in Epilepsy: What We Are Missing. JAMA Neurol, 75(8):913915.

2. Bacon, G.M. (1868). On the modes of death in epilepsy. Lancet, 91(2331):555-556.

3. Sveinsson, O., Andersson, T., Carlsson, S., Tomson, T. (2017). The incidence of SUDEP: a nationwide populationbased cohort study. Neurology, 89(2):170-177.

4. Darwin, C.R. (1872). The expression of the emotions in man and animals. London: John Murray. 1st edition.

5. Devinsky, O., Spruill, T., Thurman, D., Friedman, D. (2016). Recognizing and preventing epilepsy-related mortality: A call for action. Neurology, 86(8):779-86. 